\title{
ANACON: ANALIZADOR DE CONSULTAS SQL
}

\author{
(ANACON: SQL QUERY ANALYZER)
}

\author{
Piedad Garrido \\ Francisco Martínez \\ Jesús Tramullas \\ Universidad de Zaragoza (España) \\ Gabriel Fuertes \\ StarGlob Soluciones Informáticas (España)
}

\section{RESUMEN}

A continuación se presenta una experiencia docente en el desarrollo y uso de una herramienta de apoyo para las prácticas de la asignatura Bases de Datos I en la Escuela Universitaria Politécnica de Teruel. ANACON, es un analizador de consultas SQL que permite que los alumnos puedan aprender de forma más rápida e intuitiva a realizar consultas en dicho lenguaje, sin tener que conocer los aspectos más complejos y las particularidades que suelen presentar los analizadores de consultas que integran los SGBDR.

En este documento se presenta la herramienta, así como los resultados obtenidos al ser utilizada, en cuanto a número de presentados sobre matriculados y a las calificaciones obtenidas por los alumnos durante el curso académico 2004/2005. A pesar de que puede ser precipitado extraer conclusiones, los resultados nos animan a mejorar algunos aspectos de la aplicación, así como a continuar utilizando la herramienta en las prácticas de la asignatura.

Palabras clave: analizador de consultas, bases de datos, SQL, diseño de programas informáticos educativos.

\footnotetext{
ABSTRACT

Next, we will introduce a teaching experience about the development and application of a support tool for the practical lessons of the Database I subject at the Escuela Universitaria Politécnica de Teruel. ANACON is a query analyzer based on SQL (Structured Query Language), which allows pupils to learn
} 


\section{P. GarRido; F. Martínez; J. Tramullas; G. Fuertes \\ ANACON: ANALIZADOR DE CONSULTAS SQL}

quickly and intuitively this language without having to know the complex aspects and the special features that usually show the query analyzers built-in the DBMS (Database Management Systems).

In this article we describe the tool, just as the obtained results after making use of it, related on the one hand to the presented number over registered number and on the other hand, to the pupil marks obtained during the academic course 2004/2005. In spite of the fact that it can be soon to make conclusions, the results encourage us to improve some application features, and to carry on with the tool in the practical lessons of the subject.

Key words: query analyzer, database, SQL, educational computer programs design.

En el actual plan de estudios del título de Ingeniero Técnico en Informática de Gestión en la Universidad de Zaragoza, el estudio de la disciplina de Bases de Datos se divide en dos asignaturas de carácter troncal y obligatorio, respectivamente: Bases de Datos I y Bases de Datos II (Garrido, 2006) ubicadas en el quinto y sexto semestre de la titulación.

En la asignatura Bases de Datos I se definen conceptos tales como qué es un Sistema de Información, sus componentes y funciones, qué se entiende por SGBDR (Sistema Gestor de Bases de Datos Relacional), así como qué es una Base de Datos. Posteriormente, se presenta una panorámica del modelo relacional de datos, se habla de la organización física de las bases de datos, y se trata en profundidad el lenguaje SQL (Structured Query Language) haciendo especial hincapié en cómo se ha ido adaptando a la evolución del modelo relacional de datos.

La asignatura de Bases de Datos II básicamente es una continuación de la de Bases de Datos I. Su principal objetivo es presentar una metodología de diseño de bases de datos basada en el modelo Entidad-Relación.

La docencia de ambas asignaturas se organiza en (4+2) créditos LRU, es decir 4 créditos de teoría y problemas y 2 créditos de trabajo en el laboratorio. En la parte de teoría y problemas los alumnos reciben los conceptos teóricos más importantes de la asignatura en forma de clase magistral y realizan problemas de cada uno de los temas que se imparten. En la parte de las prácticas de laboratorio, el trabajo de los alumnos consiste en la realización, de modo coordinado con las clases de teoría y problemas, de una serie de prácticas que refuerzan la comprensión de los conceptos estudiados y permiten su aplicación.

En este artículo, se presenta la experiencia en el desarrollo y uso de una herramienta didáctica desarrollada en la Escuela y que ayuda a los alumnos en el 
aprendizaje y uso del lenguaje SQL para la recuperación de la información en Bases de Datos Relacionales. Esta aplicación, al igual que otras ya desarrolladas en el Centro, como por ejemplo, DerEditor (Garrido, 2005), va a ser próximamente distribuida de forma libre bajo licencia GNU Public License (GPL), para que esté a disposición de toda la comunidad académica.

El contenido del presente trabajo está organizado de la siguiente manera: en el siguiente apartado, se explica la experiencia docente y por qué surgió la idea del desarrollo de una herramienta de estas características. En el tercer apartado se comenta el plan de trabajo a desarrollar por parte de los alumnos durante las prácticas de laboratorio. Un cuarto apartado, mostrará la descripción de la herramienta comentando muy brevemente su funcionamiento y cómo se deben realizar las conexiones con las distintas bases de datos que se deseen utilizar. Nuestra idea es que cualquier alumno pueda descargarse y utilizar la aplicación como apoyo a su proceso de aprendizaje, de forma muy sencilla sin necesidad de tener un equipo muy potente, con total libertad y sin ningún coste, características que la hacen extremadamente útil en otros modelos de aprendizaje como la enseñanza semipresencial o no presencial, que cada vez están más presentes en nuestras universidades. Finalmente, se detallarán los resultados de la experiencia, se expondrán una serie de posibles ampliaciones y mejoras para las futuras versiones de la misma y se presentarán las conclusiones de la puesta en marcha de esta experiencia.

\section{PLANTEAMIENTO DE LA EXPERIENCIA DOCENTE}

La idea del proyecto surgió a principios del curso académico 2003/2004, debido a que en las prácticas de la asignatura Bases de Datos I, los alumnos deben realizar consultas a bases de datos relativamente sencillas, sin tener que aprender las particularidades de los distintos SGBDR.

Los problemas que presentan dichos Sistemas Gestores suelen ser por un lado, la complejidad de sus interfaces, teniendo en cuenta que los alumnos en ese momento no tienen porqué haber utilizado todavía ninguno. Por otro lado, el tamaño necesario para ser instalados debido a que incorporan muchas funcionalidades que no son necesarias para realizar las prácticas de esta asignatura y finalmente, la licencia con la que se distribuyen, ya que muchas son de pago.

Desarrollar una aplicación muy sencilla, que cumpliera con los requerimientos mínimos para realizar las prácticas y que pudiera ser distribuida de forma libre, simplificaría esos problemas y reduciría el tiempo necesario para el aprendizaje del 
uso del analizador de consultas por parte de los alumnos, permitiendo que se ciñan al estándar y funcionen bajo cualquier SGBD.

Además, proponiendo el desarrollo de la herramienta como Trabajo Final de Carrera, se continuaría con la idea de que los alumnos realicen trabajos relacionados directamente con la disciplina, se interesen en los desarrollos de Software Libre, y que esas aplicaciones sean útiles para el resto de sus compañeros y de la comunidad educativa. Cabe destacar que los alumnos han mostrado un especial interés por este último aspecto.

Por otra parte, como valor añadido se puede realizar el desarrollo teniendo en cuenta la experiencia directa del alumno que ya ha realizado las prácticas con los problemas y vicisitudes que tuvo en su momento, aportando soluciones y nuevas ideas para mejorarlas. En nuestra Escuela, y especialmente relacionado con las Bases de Datos, ya ha habido algún otro ejemplo (Garrido, 2006). La idea inicial era la de utilizar la aplicación como una herramienta didáctica complementaria para facilitar el aprendizaje de los alumnos en los posteriores cursos académicos.

Para poner en marcha el plan, se decidió utilizarla únicamente en uno de los dos grupos de prácticas, a modo de experiencia piloto. Con el otro grupo de prácticas pareció adecuado seguir realizando las prácticas con las aplicaciones tal y como se había hecho hasta entonces, y así poder comparar.

Para el curso académico 2005/2006, el grupo A utilizaría ANACON y los alumnos del grupo B utilizarían los analizadores de consultas de Microsoft Access, Microsoft SQL Server 2000 y Oracle.

El modo de asignación a los grupos ha sido muy sencillo, ya que la parte práctica de la asignatura de Bases de Datos I está organizada en dos grupos: el A y B. Las sesiones tienen carácter quincenal, por lo que hay dos sesiones distintas de la misma práctica y en el mismo horario. Los alumnos se apuntan vía on-line de forma voluntaria a los grupos de prácticas, por lo que el cupo de cada grupo se fija en el 50\% de los matriculados.

Una vez presentado el escenario de la experiencia docente, posteriormente se presentará en el apartado de resultados, los datos obtenidos después de su implantación en el primer cuatrimestre del curso académico 2005/2006. Para ello, se hará un seguimiento de los alumnos de ambos grupos basado en algunos indicadores de vital importancia para poder sacar unas conclusiones objetivas sobre el uso de 
la herramienta construida y su utilidad. De esta manera, se mostrará el índice de entrega de prácticas por parte de los alumnos, el índice de estudiantes aprobados, y la nota final de la asignatura.

\section{Trabajo del alumno}

La parte práctica de la asignatura Bases de Datos I estaba organizada, para los anteriores cursos académicos antes de la puesta en marcha del uso de la nueva herramienta ANACON, tal y como aparece en la Figura 1. En dicha figura, aparece desglosado por sesiones el calendario de prácticas propuesto inicialmente.

Para poder utilizar la nueva herramienta, se decidió que para ambos grupos, las prácticas 1,3 y 5 serían completamente idénticas. Sin embargo, en el grupo piloto de la experiencia, para las prácticas 2 y 4 se utilizaría específicamente ANACON, en lugar del analizador de consultas de Access 2002 y el analizador de consultas de SQL Server 2000.

Cabe destacar que el tiempo dedicado al aprendizaje del funcionamiento de ANACON fue menor, que el tiempo dedicado al aprendizaje del funcionamiento de los otros analizadores de consultas de los gestores de bases de datos comentados anteriormente, debido sobretodo a la sencillez de uso en comparación con éstos.

De este modo, el número promedio de consultas resueltas por parte de los alumnos del grupo A fue más elevado, de hecho hubo que dejar disponible un bloque de consultas adicional y no contemplado en la programación inicial de las prácticas.

Esto corrobora la ventaja de disminuir el tiempo necesario para familiarizarse con un determinado entorno. Cuanto más sencillo sea, más tiempo se podrá dedicar a realizar realmente ejercicios prácticos.

Ya que ANACON es un analizador puro y duro de consultas SQL, el alumno puede poner en práctica enseguida los conocimientos aprendidos en las clases teóricas de la asignatura. Sin embargo, el uso de cualquier analizador de consultas integrado en cualquier sistema gestor de base de datos comercial, ya sea propietario o libre, pasa por el conocimiento de algunos aspectos como las aplicaciones integrantes del conjunto de programas, del administrador corporativo, etc., tareas normalmente más complicadas y que el alumno no tiene porqué conocer ya que no son objetivo de esta asignatura. 


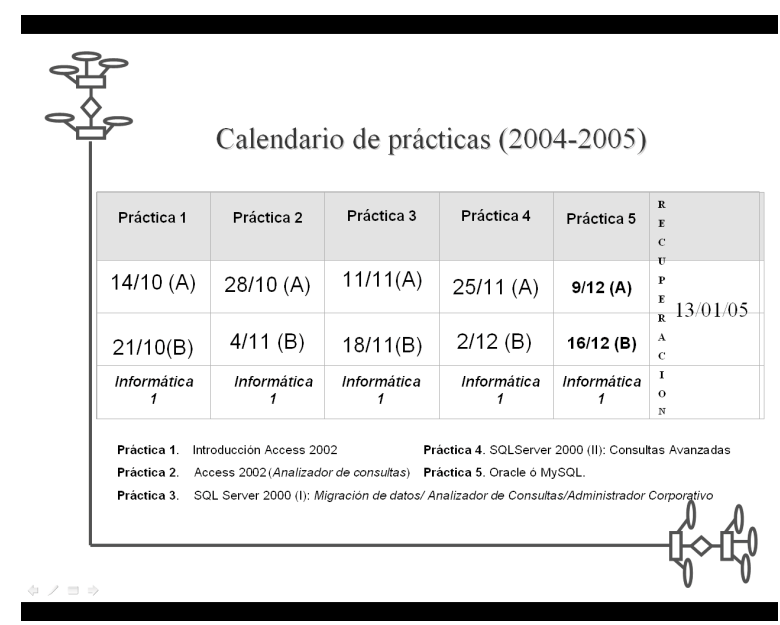

Figura 1. Calendario de prácticas de la asignatura.

\section{DESCRIPCIÓN DE LA HERRAMIENTA ANACON}

Antes de comenzar con el desarrollo, la primera tarea a llevar a cabo, era la de buscar, probar y analizar el software de características similares que ya existiera.

Después de instalar y probar herramientas como WinSQL, WinRDBI, Active Visual Query Builder y Query Tool, se llegó a la conclusión de que no cumplían exactamente con los requisitos establecidos inicialmente. También se intentó ver las distintas características que tienen en común y los posibles defectos que presentan. Otro detalle a tener en cuenta es que todos ellos se distribuyen en inglés, por lo que se echa de menos una herramienta de este tipo para que pueda ser usada por la comunidad hispano parlante.

Aunque no es lo más importante, comentar que la herramienta ha sido desarrollada con Delphi, principalmente debido a que el alumno proyectista encargado de desarrollar la aplicación, una vez realizado el análisis y el diseño, y teniendo en cuenta que durante la carrera ya había programado en otros lenguajes como ADA, Java o C, decidió utilizar otro lenguaje y entorno de programación nuevo.

Para facilitar la instalación por parte de los usuarios, se ha creado un instalador construido con el NSIS (Nullsoft Scriptable System). Durante el proceso de instalación se indica el tipo de licencia con la que se distribuye la aplicación que es la GPL, tal y como se ha indicado anteriormente. El proceso de desinstalación del 
producto también es una tarea muy sencilla. A continuación, se va a describir cómo se almacenan y cómo se pueden generar las distintas conexiones con las bases de datos a las que se desea consultar.

\section{Conexión con las bases de datos}

ANACON permite conectarse con la gran mayoría de los Sistemas Gestores de Bases de Datos, de forma muy sencilla. Esto la hace independiente y distinta a la gran mayoría de Analizadores de Consultas.

Para poder crear una nueva conexión a una base de datos determinada, únicamente se deberá crear un fichero con extensión .udl, que no es sino la extensión de los archivos de vínculos a datos de Microsoft. Al hacer doble clic sobre el fichero, se abrirá una pantalla desde la que se podrá seleccionar un driver. A continuación habrá que proporcionar la ruta en la que se encuentra ubicada la base de datos, así como el login y el password del usuario o grupo de usuarios, siempre y cuando fuera necesario. Una vez generado el archivo .udl se podrá realizar la conexión con la base de datos y por tanto, diseñar cualquier consulta y lanzarla contra el repositorio de información que se haya escogido. Para cada base de datos distinta, se tendrá que tener un archivo de vínculo a datos distinto.

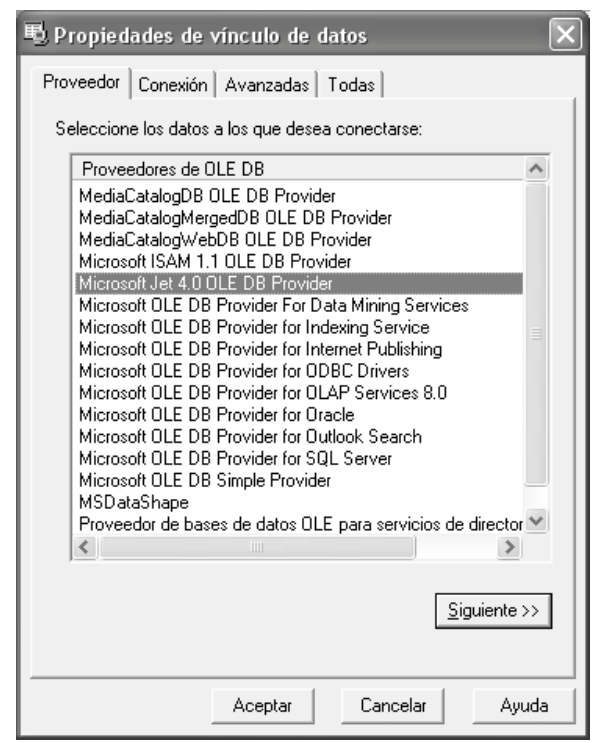

Figura 2. Ejemplo de configuración de una nueva conexión. 


\section{P. GarRido; F. Martínez; J. Tramullas; G. Fuertes \\ ANACON: ANALIZADOR DE CONSULTAS SQL}

\section{Funcionamiento}

Una vez creados los archivos necesarios para llevar a cabo las conexiones a las distintas bases de datos que se desea consultar, ya se está en disposición de ejecutar la aplicación y poder trabajar con ella. El uso de la aplicación es extremadamente sencillo e intuitivo. En la Figura 3, puede verse la interfaz de la aplicación.

La pantalla se divide en varios cuadros de texto. En la parte izquierda de la misma aparece el nombre de la base de datos a la que se ha conectado, las tablas que posee, así como en el momento que se seleccione una, los atributos de la tabla.

En la parte inferior izquierda, además aparece un cuadro explicativo que irá comentando en cada caso y de manera escueta la acción que realizará la consulta que se vaya a ejecutar. Este cuadro es especialmente útil para los alumnos cuando empiezan a practicar con las consultas de selección con SQL.

Para tener una información más amplia sobre cualquier tipo de consulta que soporte el sistema, o simplemente sobre el funcionamiento de la aplicación, se puede acceder a la Ayuda que posee la aplicación.

El cuadro principal de la interfaz, al que se le ha concedido un mayor tamaño, es el destinado a mostrar los resultados de las consultas realizadas, lo que permite a los alumnos comprobar si la consulta realizada en un determinado momento ha devuelto los resultados esperados y por tanto la da por válida y puede pasar a realizar la siguiente. Las diferentes consultas deberán ser introducidas por el usuario en el cuadro de texto Consultas, situado en la parte inferior.

La aplicación también posee un sencillo menú de opciones y una barra de herramientas con las distintas acciones que se pueden llevar a cabo, tales como conectar con una base de datos, realizar una nueva consulta, cargar una consulta previamente almacenada, guardar las consultas, ejecutarlas, etc.

Una vez que se ha conectado con la base de datos elegida, ya se pueden introducir y ejecutar las diferentes consultas que se desee y ver los resultados de las mismas, o los posibles errores. 


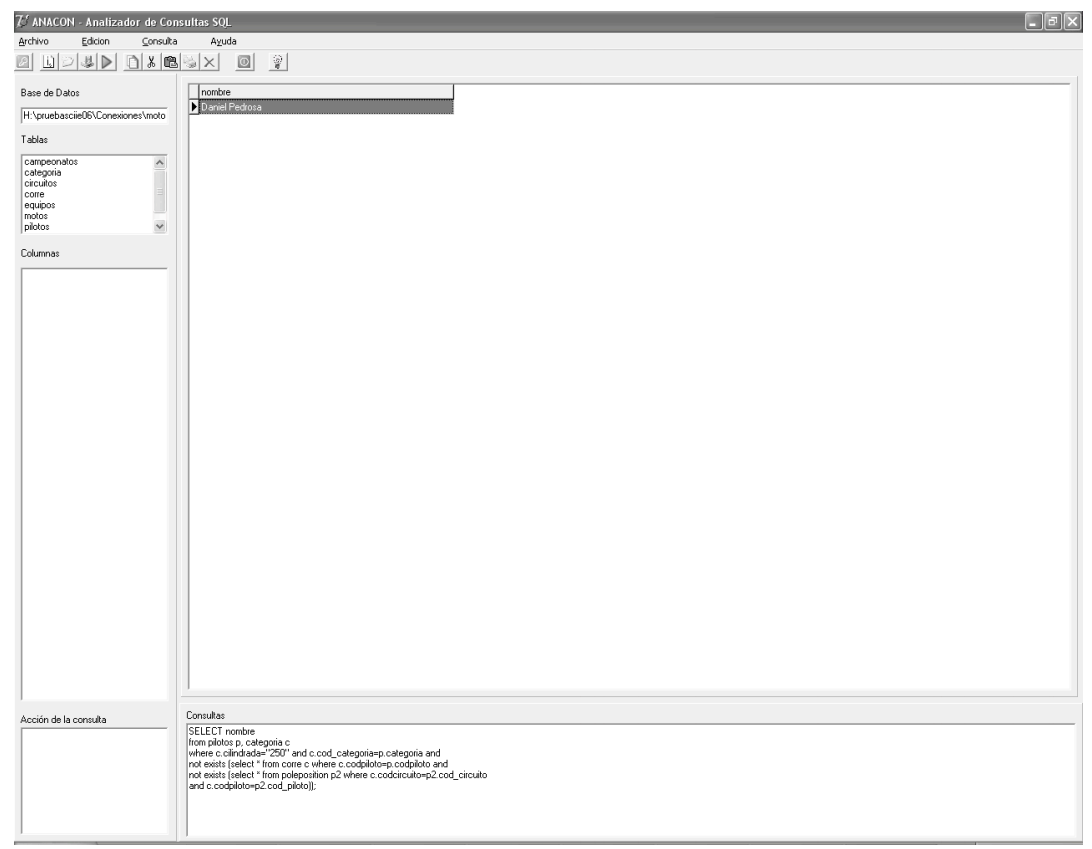

Figura 3. Interfaz de ANACON.

\section{RESULTADOS}

Tal y como se comentó en el apartado del planteamiento de la experiencia docente, una vez finalizado el primer cuatrimestre del curso académico 2005/2006, se procedió a la recogida de los datos destacados con anterioridad, tanto para el grupo A de prácticas (piloto) como para el grupo B, obteniendo los resultados que se muestran en las tablas que aparecen a continuación.

\begin{tabular}{|l|c|c|}
\hline \multicolumn{3}{|c|}{ CURSO 2005/2006 } \\
\hline & Entregan prácticas & Nota Media (Prácticas) \\
\hline Grupo A & & 7,8 \\
\hline 12 alumnos & 10 & \\
\hline Grupo B & & 7,1 \\
\hline 12 alumnos & 8 & \\
\hline
\end{tabular}

Tabla 1. Resultados de las prácticas de Laboratorio al final del cuatrimestre. 


\section{P. Garrido; F. Martínez; J. Tramullas; G. Fuertes ANACON: ANALIZADOR DE CONSULTAS SQL}

Se decidió realizar la experiencia piloto únicamente sobre el grupo A, intentando comparar directamente las sensaciones y reacciones de los alumnos de los dos grupos.

A pesar de que los resultados no son rotundos, como veremos, se ha decidido utilizar ANACON para los dos grupos de prácticas a partir del curso académico $2006 / 2007$.

La nota media de las prácticas puede no ser el dato más representativo. Además puede verse afectada por muchos otros factores externos que pueden influir en la misma, como la carga de trabajo de otras asignaturas, el grado de madurez, el ambiente del grupo de prácticas, etc. Salvando estos detalles, nosotros sí que consideramos que puede observarse una pequeña tendencia positiva, que nos anima a continuar con su uso.

Otro aspecto importante a tener en cuenta es el seguimiento del alumnado en cuanto al índice de presentados en las convocatorias de exámenes y al porcentaje de aprobados en la asignatura.

Hay que tener en cuenta que el examen se hace en formato papel en un aula convencional (no informatizada), el diseño de las consultas en SQL hay que hacerlo conforme al estándar y sin disponer de un analizador de consultas que interprete la solución e indique al alumno si lo que está haciendo está bien o mal.

Los datos que aparecen en la Tabla 2 muestran una ligera mejora en el porcentaje de alumnos que se presentan y superan la asignatura en este último curso, en el que se ha utilizado ANACON en el grupo A de prácticas. Hay que destacar que los datos que aparecen para ambos cursos son los relativos a la primera convocatoria, pues en el curso 05/06 es la única que se había realizado hasta el momento (también en la Tabla 3).

\begin{tabular}{|c|c|c|c|}
\cline { 2 - 4 } \multicolumn{1}{c|}{} & APROBADO & SUSPENSO & N.P. \\
\hline 04/o5 & & & \\
\hline Grupo A & 9 & 3 & 1 \\
\hline Grupo B & 8 & 5 & 2 \\
\hline 05/o6 & & & \\
\hline Grupo A & 10 & 2 & 1 \\
\hline Grupo B & 8 & 2 & 1 \\
\hline
\end{tabular}

Tabla 2. Notas de la primera convocatoria de los dos últimos cursos. 


\section{P. Garrido; F. MartíneZ; J. Tramullas; G. Fuertes ANACON: ANALIZADOR DE CONSULTAS SQL}

Lo que también se ha observado con respecto a otros años, es que el número de suspensos ha descendido. Se piensa que es un dato positivo que nos anima a tomar varias medidas como utilizar la herramienta ANACON en ambos grupos de prácticas, a realizar parte del examen de la asignatura en el laboratorio, e incluso a incentivar su uso en otras asignaturas también relacionadas con las Bases de Datos, pero que se imparten en otras titulaciones distintas a la ingeniería de Informática.

\begin{tabular}{|c|c|c|c|}
\cline { 2 - 4 } \multicolumn{1}{c|}{} & APROBADO & SUSPENSO & N.P. \\
\hline 01/o2 & 11 & 3 & 2 \\
\hline $02 / 03$ & 10 & 13 & 3 \\
\hline $03 / 04$ & 32 & 12 & 3 \\
\hline 04/05 & 20 & 6 & 2 \\
\hline 05/o6 & 18 & 4 & 2 \\
\hline
\end{tabular}

Tabla 3. Resultados de la asignatura en los últimos cinco años.

\section{AMPLIACIONES Y MEJORAS}

A continuación se presentan algunas de las diferentes mejoras que se podrían introducir en la aplicación ANACON, si bien hay que tener en cuenta que habría que anteponer la sencillez de la aplicación antes que añadir demasiadas funcionalidades, pues la sencillez es quizá la característica que la hace más adecuada y atractiva que otras aplicaciones ya existentes.

Las posibles ampliaciones que se podrían contemplar en esta aplicación serían por una lado, añadir la posibilidad de ejecutar cualquier consulta de tipo DDL (Database Definition Language, o Lenguaje de Definición de Bases de Datos). Estas consultas, como bien es sabido, permiten realizar operaciones de inserción, borrado y modificación de las tuplas de cada una de las tablas que forman cualquier base de datos. Este tipo de consultas, también son estudiadas en las clases de teoría y problemas de la asignatura de Bases de Datos I.

Por otro lado, también se podría implementar una opción que permitiera ver cómo está diseñada la base de datos que se desea consultar en ese momento, ya sea mediante texto, si se muestra el esquema lógico, o gráficamente mostrando el esquema conceptual. 
De esta forma, se conseguiría que las consultas fueran realizadas de forma más rápida por parte de los alumnos, pudiendo invertir el tiempo en realizar un número mayor de consultas.

Este aspecto sin embargo, no está del todo claro, pues el tiempo que dedica el alumno a la construcción en papel de las tablas, sus atributos y sus relaciones, también es beneficioso pues le permite asentar los conocimientos adquiridos y detectar las posibles dudas que pueda tener.

Finalmente, y si no sólo se tuviera en cuenta el modelo de datos relacional, se podría mejorar la aplicación para que pudiera acceder no sólo a bases de datos relacionales, sino también a bases de datos documentales, bases de datos orientadas a objetos, e incluso bases de datos nativas en XML.

Como tarea a realizar en breve plazo se tiene también la intención de distribuir la aplicación de forma libre en sourceforge.net, al igual que ya se ha hecho con otras aplicaciones desarrolladas con anterioridad.

\section{CONCLUSIONES}

En el presente artículo se presenta, por un lado, el desarrollo de una herramienta que puede ser utilizada para facilitar el proceso de aprendizaje de los alumnos de asignaturas básicas relacionadas con las Bases de Datos. La idea es que el manejo de la nueva herramienta sea lo más sencillo posible, y que sea independiente y estándar, es decir que no presente aspectos directamente relacionados con alguno de los Sistemas Gestores de Bases de Datos existentes en el mercado.

Por otro lado, se comenta la experiencia puesta en marcha en el presente curso académico, al usar dicha aplicación en uno de los grupos de prácticas, y se presentan los resultados obtenidos que parecen esperanzadores, lo que sugiere que sea utilizada en las posteriores ediciones de la asignatura y que sea distribuida para que pueda ser usada por el resto de la comunidad universitaria.

En cuanto a las ampliaciones o mejoras, cabe destacar que hay que actuar con bastante cautela pues una de las principales armas de esta aplicación es su extremada sencillez. Teniendo en cuenta en primer lugar, el escaso número de alumnos que últimamente acceden a las distintas titulaciones de la Universidad, en segundo lugar, que está cambiando el perfil de los alumnos, pues muchos ya están trabajando, y finalmente los nuevos planes de estudio y las nuevas metodologías docentes, se cree 
que el desarrollo y uso de herramientas como ANACON, muy sencillas y pensadas para que los alumnos puedan aprender rápidamente y de forma fácil, va a extenderse en los próximos años.

Esta aplicación también estará próximamente disponible para todos los alumnos, especialmente los que realicen sus estudios de forma semipresencial, a través del ADD (Anillo Digital Docente de la Universidad de Zaragoza).

Para finalizar, apuntar que se está valorando la posibilidad de usarla como una herramienta de evaluación. La idea es que el alumnado pueda realizar el examen en el laboratorio, y así probar las consultas que hasta el momento está realizando en formato papel.

\section{REFERENCIAS BIBLIOGRÁFICAS}

Active Visual Query Builder. [en línea]. Disponible en: http://www. activequerybuilder.com/ [consulta 2006, 20 de Junio]

Anillo Digital Docente de la Universidad de Zaragoza. [en línea]. Disponible en: http://add.unizar.es [consulta 2006, 20 de Junio]

Beaulieu, A. (2005). Learning SQL. O'Reilly.

Cantu, M. (2003). La Biblia de Delphi. Madrid: Anaya.

Celma, M.; Casamayor, J. C.; Mota, L. (2003). Bases de Datos Relacionales. Madrid: Prentice Hall.

Charte, F. (2003). Delphi 7.0 y Kylix 3.o. Madrid: Anaya.

Date, C. J. (2001). Introducción a los sistemas de Bases de Datos. Madrid: Prentice Hall.

DerEditor. [en línea]. Disponible en: http:// dereditor.sourceforge.net/ [consulta 2006, 20 de Junio]

Elmasri, R. A.; Navathe, S. B. (2002). Fundamentos de Sistemas de Bases de Datos. Addison Wesley.

Garrido, P. (2006). Página web de la asignatura Base de Datos I. [en línea].
Disponible en: http://eupt2.unizar.es/ bd1 [consulta 2006, 20 de Junio]

Garrido, P. (2006). Página web de la asignatura Base de Datos II. [en línea]. Disponible en: http://eupt2.unizar.es/ $\underline{\mathrm{bd} 2}$ [consulta 2006, 20 de Junio]

Garrido, P.; Coll, M.; Martínez, F. J. (2005). DerEditor4GL: Software para la docencia en el diseño de Bases de Datos. Actas de las XI Jornadas de Enseñanza Universitaria de la Informática. Madrid: Thomson-Paraninfo.

Groff, J. R.; Weinberg, P. N. (2003). SQL: Manual de Referencia. Mc-Graw Hill.

Gutiérrez, J. J.; Escalona, M. J.; Villadiego, D.; Mejías, M. (2005). Comparativa de Herramientas para la Enseñanza de Lenguajes Relacionales. Actas de las XI Jornadas de Enseñanza Universitaria de la Informática. Madrid: ThomsonParaninfo.

Molinaro, A. (2005). SQL CookBook. O'Reilly.

NullSoft Scriptable Install System. [en línea]. Disponible en: http://nsis. sourceforge.net/ [consulta 2006, $20 \mathrm{de}$ Junio]

Potnia. [en línea]. Disponible en: http:// 
potnia.sourceforge.net/] [consulta 2006, 20 de Junio]

Query Tool. [en línea]. Disponible en: http://www.querytool.com/ [consulta 2006, 20 de Junio]

Ullman, J. D.; Widom, J. (1999). Introducción a los sistemas de Bases de Datos. Madrid: Pearson.
WinRDBI Educational Tool. [en línea]. Disponible en: http://www.eas.asu. edu/ winrdbi/ [consulta 2006, 20 de Junio]

WinSQL .[en línea]. Disponible en: http://www.synametrics.com/ SynametricsWebApp/WinSQL.jsp [consulta 2006, 20 de Junio]

\section{PERFIL ACADÉMICO Y PROFESIONAL DE LOS AUTORES}

Piedad Garrido Picazo, profesora del Departamento de Informática e Ingeniería de Sistemas de la Universidad de Zaragoza. Miembro del Grupo de Educación Digital de dicha universidad, actualmente es la investigadora responsable del proyecto de innovación educativa “TORICO” que versa sobre el análisis, diseño e implementación de una herramienta de trabajo colaborativo multidisciplinar e interdepartamental.

E-mail:piedad@unizar.es

Gabriel Fuertes, uno de los socios fundadores de la empresa StarGlob Soluciones Informáticas. Empresa creada por profesionales con amplia experiencia en las Tecnologías de la Información que se dedica a tareas de asesoramiento y desarrollo de productos en los siguientes campos:

Desarrollo de Software a Medida

Diseño y Creación de Páginas Web

$\mathrm{I}+\mathrm{D}+\mathrm{I}$

Mantenimientos Informáticos a Empresas

Consultoría / Asesoría Informática

Formación en las Nuevas Tecnologías

E-mail: gabi@starglob.com

Francisco J. Martínez Domínguez, profesor del Departamento de Informática e Ingeniería de Sistemas. Actualmente es miembro del Proyecto de Innovación Educativa "TORICO" y ha participado en el análisis, diseño e implementación de aplicaciones educativas, desarrolladas con software libre, para la adaptación de algunas asignaturas de la titulación de ITIG al Espacio Europeo de Educación Superior.

E-mail: f.martinez@unizar.es 
Jesús Tramullas, profesor titular de Documentación Automatizada en la Universidad de Zaragoza. Su actividad docente e investigadora se centra en las áreas de:

Software libre para servicios de información digital

Auditoría de información

Gestión de contenidos

Repositorios digitales

Bibliotecas digitales

Gestión del conocimiento y de la información

Arquitectura de la información y usabilidad

E-mail: jesus@tramullas.com

DIRECCIÓN DE LOS AUTORES

Universidad de Zaragoza

Ciudad Escolar s/n

44003 Teruel (España)

Fecha de recepción del artículo: 19/10/06

Fecha de aceptación del artículo: 13/02/07 